Discrete Comput Geom 30:65-85 (2003)

DOI: $10.1007 / \mathrm{s} 00454-003-2922-9$

\title{
Different Bounds on the Different Betti Numbers of Semi-Algebraic Sets*
}

\author{
Saugata Basu \\ School of Mathematics and College of Computing, \\ Georgia Institute of Technology, \\ Atlanta, GA 30332, USA \\ saugata@math.gatech.edu
}

\begin{abstract}
A classic result in real algebraic geometry due to Oleinik-Petrovskii, Thom and Milnor, bounds the topological complexity (the sum of the Betti numbers) of basic semi-algebraic sets. This bound is tight as one can construct examples having that many connected components. However, till now no significantly better bounds were known on the individual higher Betti numbers.

We prove better bounds on the individual Betti numbers of basic semi-algebraic sets, as well as arrangements of algebraic hypersurfaces. As a corollary we obtain a polynomial bound on the highest Betti numbers of basic semi-algebraic sets defined by quadratic inequalities.
\end{abstract}

\section{Introduction}

The combinatorial, algebraic and topological analysis of arrangements of real algebraic hypersurfaces in higher dimensions are active areas of research in computational geometry (see [1], [11] and [18]). Arrangements of lines and hyperplanes have been studied quite extensively earlier. It was later realized that arrangements of curved surfaces are a significant generalization and have a wider range of applications. Many geometric problems reduce to problems involving such arrangements, such as motion planning for a robot with several degrees of freedom, generalized Voronoi diagrams where the Voronoi cells need not be polyhedral, geometric problems involving transversals, and many problems in geometric optimization. All such problems can be rephrased in terms of certain substructures (e.g., single cell, lower/upper envelopes, etc.) of an arrangement of surfaces in higher dimensions. There has been much progress in analyzing the

* This research was supported in part by NSF Grant CCR-0049070 and an NSF Career Award 0133597. 
combinatorial complexity - that is, the number of cells (appropriately defined) of various dimensions occurring in the boundary—of substructures in arrangements [18].

However, there is another source of geometric complexity in arrangements of hypersurfaces-namely, topological complexity. Arrangements of hypersurfaces are distinguished from arrangements of hyperplanes by the fact that arrangements of hypersurfaces are topologically more complicated than arrangements of hyperplanes. For instance, a single hypersurface or intersections of two or more hypersurfaces, can have non-vanishing higher homology groups and thus sets defined in terms of such hypersurfaces can be topologically more complicated in various non-intuitive ways. It is often necessary to estimate the topological complexity of arrangements [9] and sometimes these estimates even play a role in bounding the combinatorial complexity, see [4].

An important measure of the topological complexity of a set $S$ is the Betti numbers $b_{i}(S)$. Here and elsewhere in the paper the set $S$ will always be semi-algebraic (that is, defined in terms of a finite number of real polynomial equalities and inequalities) and closed and $b_{i}(S)$ will denote the rank of the $H^{i}(S)$ (the $i$ th singular cohomology group with real coefficients). Intuitively, $b_{i}(S)$ measures the number of $i$-dimensional holes in $S$. The zeroth Betti number $b_{0}(S)$ is the number of connected components.

For example, if $T$ is topologically a hollow torus, then $b_{0}(T)=1, b_{1}(T)=2, b_{2}(T)$ $=1, b_{i}(T)=0, i>2$, confirming our intuition that the torus has two one-dimensional holes and one two-dimensional hole. Analogously, for the two-dimensional sphere, $S$, $b_{0}(S)=1, b_{1}(S)=0, b_{2}(S)=1, b_{i}(S)=0, i>2$.

The basic result in bounding the Betti numbers of semi-algebraic sets defined by polynomial inequalities was proved independently by Oleinik and Petrovskii [15], Thom [19] and Milnor [14].

They proved:

Theorem 1 [15], [19], [14]. Let $S \subset R^{k}$ be the set defined by the conjunction of $n$ inequalities,

$$
\begin{aligned}
P_{1} \geq 0, \ldots, P_{n} \geq 0, & P_{i} \in R\left[X_{1}, \ldots, X_{k}\right] \\
\operatorname{deg}\left(P_{i}\right) \leq d, & 1 \leq i \leq n .
\end{aligned}
$$

Then

$$
\sum_{i} b_{i}(S)=O(n d)^{k}
$$

The above bound is actually quite tight. One can see this by considering the following example: Let

$$
P_{i}=L_{i, 1}^{2} \cdots L_{i,\lfloor d / 2\rfloor}^{2}-\varepsilon,
$$

where the $L_{i j}$ 's are generic linear polynomials and $\varepsilon>0$ and sufficiently small. The set $S$ defined by $P_{1} \geq 0, \ldots, P_{n} \geq 0$ has $\Omega(n d)^{k}$ connected components and hence $b_{0}(S)=\Omega(n d)^{k}$.

However, one fails to construct examples such that $b_{i}(S)=\Omega(n d)^{k}$ for $i>0$. Thus, one is led to believe that tighter bounds can be proved for the higher Betti numbers. The proof technique used for proving the above result (see [14]) first replaces the semialgebraic set $S$ by another set bounded by a smooth algebraic hypersurface of degree $n d$ 
having the same homotopy type as $S$. It then proceeds to bound the Betti numbers of this hypersurface using Morse theory and Bezout's bound on the number of solutions of a system of polynomial equations. This technique does not allow one to prove separate bounds on the individual Betti numbers smaller than the general bound.

The first attempt in trying to prove better bounds for higher Betti numbers was made in [4]. It was motivated by the long-standing problem in computational geometry of bounding the combinatorial complexity of a single cell in an arrangement of surface patches. The following result was proved in [4] which bounds the higher Betti numbers of a single connected component of a basic semi-algebraic set.

Theorem 2 [4]. Let $C \subset R^{k}$ be a connected component of the set $S$ defined by the conjunction of $n$ inequalities,

$$
\begin{aligned}
P_{1} \geq 0, \ldots, P_{n} \geq 0, & P_{i} \in R\left[X_{1}, \ldots, X_{k}\right], \\
\operatorname{deg}\left(P_{i}\right) \leq d, & 1 \leq i \leq n .
\end{aligned}
$$

Then

$$
\sum_{i} b_{i}(C)=\left(\begin{array}{c}
n \\
k-1
\end{array}\right) O(d)^{k}
$$

The proof of the above result involved Morse theory of stratified spaces in an essential way. The technique used to prove this result was also crucial in extending the method developed by Halperin and Sharir [12] for bounding the combinatorial complexity of a single cell in an arrangement of surface patches to higher dimensions (see [4] for more details).

\section{Results}

The main contribution of this paper is a generalization of Theorem 2. We show that it is possible to obtain a "graded" bound on the Betti numbers of a basic semi-algebraic set. We denote the set of real zeros of a polynomial $P \in R\left[X_{1}, \ldots, X_{k}\right]$ by $Z(P)$. We prove the following theorem.

Theorem 3. Let $R$ be a real closed field and let $S \subset R^{k}$ be the set defined by the conjunction of $n$ inequalities,

$$
P_{1} \geq 0, \ldots, P_{n} \geq 0,
$$

$P_{i} \in R\left[X_{1}, \ldots, X_{k}\right], \operatorname{deg}\left(P_{i}\right) \leq d, 1 \leq i \leq n$, contained in a variety $Z(Q)$ of real dimension $k^{\prime}$ with $\operatorname{deg}(Q) \leq d$. Then

$$
b_{i}(S) \leq \sum_{j=0}^{k^{\prime}-i}\left(\begin{array}{l}
n \\
j
\end{array}\right) 2^{j+1} d(2 d-1)^{k-1} \leq\left(\begin{array}{c}
n \\
k^{\prime}-i
\end{array}\right) O(d)^{k} .
$$


One should compare the above theorem to the main results in [6] and [3] where similar bounds are proved on the number of connected components and the sum of the Betti numbers of more general (not just basic) semi-algebraic sets contained in a variety.

The combinatorial part (the part depending on $n$ ) in the above bound is tight, as one can easily construct examples such that the bound on $b_{i}$ is actually realized (see [4]). To our knowledge this is also the first bound on the Betti numbers of semi-algebraic sets that bounds the individual Betti numbers rather than their sum.

In many applications in computational geometry one is often interested in understanding the topological complexity of the whole arrangement. For instance, unions of balls in $R^{3}$ has been studied by Edelsbrunner [9] from both combinatorial and topological viewpoints motivated by applications in molecular biology, and efficient algorithms for computing the various Betti numbers of such unions are currently being studied [10]. There is also a whole body of mathematical literature studying the topology of arrangements of hyperplanes in complex as well as real spaces (see [16]).

In some simple situations the Betti numbers of a union of $n$ sets are easy to estimate. For instance, when the sets are compact and convex, a classical result of topology, the nerve lemma [17], gives us a bound on the individual Betti numbers of the union. The nerve lemma states that the homology groups of such a union is isomorphic to the homology groups of a combinatorially defined simplicial complex, the nerve complex (Fig. 1). The nerve complex has $n$ vertices and thus the $i$ th Betti number is bounded by $\left(\begin{array}{c}n \\ i+1\end{array}\right)$. (Actually, the nerve lemma requires only that all finite intersections of the sets be topologically trivial and convex sets clearly satisfy this condition.) However, when the given sets are not necessarily convex, which would be the case in many applications, the nerve lemma does not apply, and there is no a priori bound on the Betti numbers of the

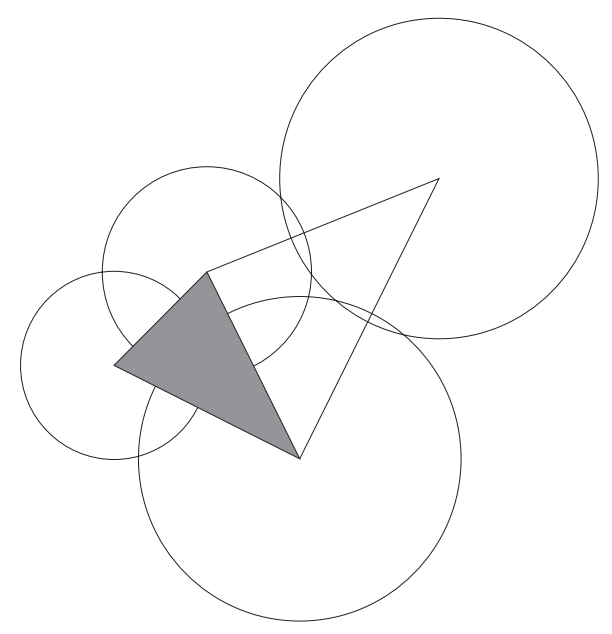

Fig. 1. The nerve complex of a union of disks. 
union. We prove the following bound dual to Theorem 3:

Theorem 4. Let $R$ be a real closed field and let $S \subset R^{k}$ be the set defined by the disjunction of $n$ inequalities,

$$
P_{1} \geq 0, \ldots, P_{n} \geq 0
$$

$P_{i} \in R\left[X_{1}, \ldots, X_{k}\right], \operatorname{deg}\left(P_{i}\right) \leq d, 1 \leq i \leq n$. Then

$$
b_{i}(S) \leq \sum_{j=0}^{i+1}\left(\begin{array}{c}
n \\
j
\end{array}\right) 3^{j} d(2 d-1)^{k-1} \leq\left(\begin{array}{c}
n \\
i+1
\end{array}\right) O(d)^{k} .
$$

An interesting consequence of our techniques is a polynomial bound on the highest Betti numbers of basic semi-algebraic sets defined by quadratic inequalities. Such sets often arise in practice, for instance, as configuration spaces of sets of points with pairwise distance constraints. They are also interesting because quadratic constraints are the simplest non-linear constraints that are topologically non-trivial.

Barvinok [2] proved the following bound on the sum of the Betti numbers of semialgebraic sets defined by a conjunction of quadratic inequalities.

Theorem 5 [2]. Let $S \subset R^{k}$ be defined by

$$
P_{1} \geq 0, \ldots, P_{\ell} \geq 0
$$

$\operatorname{deg}\left(P_{i}\right) \leq 2,1 \leq i \leq \ell$. Then the sum of the Betti numbers of $S$ is bounded above by $k^{O(\ell)}$.

Notice that unlike the bounds described previously, this bound is exponential in the number of constraints. However, if the number of constraints, $\ell$, is fixed the bound is polynomial in the dimension $k$. Thus, it gives a polynomial bound on the sum of the Betti numbers of a basic semi-algebraic set defined by few quadratic inequalities. We prove a polynomial bound on the highest Betti numbers of a basic semi-algebraic set defined by quadratic inequalities. More precisely,

Theorem 6. Let $\ell$ be any fixed number and let $R$ be a real closed field. Let $S \subset R^{k}$ be defined by

$$
P_{1} \geq 0, \ldots, P_{n} \geq 0
$$

with $\operatorname{deg}\left(P_{i}\right) \leq 2$. Then

$$
b_{k-\ell}(S) \leq\left(\begin{array}{l}
n \\
\ell
\end{array}\right) k^{O(\ell)}
$$

Notice that for fixed $\ell$ this gives a polynomial bound on the highest $\ell$ Betti numbers of $S$. Also, notice that the lowest Betti numbers of $S$ cannot be polynomially bounded. For instance, let $S$ be defined by $X_{1}\left(X_{1}-1\right) \geq 0, \ldots, X_{k}\left(X_{k}-1\right) \geq 0$. Clearly, $b_{0}(S)=2^{k}$. 
Finally, observe that similar bounds do not hold for sets defined by polynomials of degree greater than two. For instance, the set defined by the single quartic equation,

$$
\sum_{i=1}^{k} X_{i}^{2}\left(X_{i}-1\right)^{2}-\varepsilon=0,
$$

will have $b_{k-1}=2^{k}$, for small enough $\varepsilon>0$.

Our technique for proving these bounds involves using certain spectral sequences related to the generalized Mayer-Vietoris exact sequence ([13] is a good reference). The spectral sequence method also has algorithmic consequences for designing efficient algorithms for computing the Betti numbers of a set defined by polynomial inequalities. Results in this direction are reported in a forthcoming paper [5].

The rest of the paper is organized as follows. In Section 3 we briefly discuss some well-known constructions in algebraic topology, namely, double complexes and their associated spectral sequences. In Section 4 we prove the theorems stated above.

\section{Mathematical Preliminaries}

Our method of proving Theorems 3 and 4 goes via the theory of spectral sequences related to a generalized Mayer-Vietoris sequence. We give a brief review of the relevant notions below (see [13] for more details). Note that closed and bounded semi-algebraic sets are finitely triangulable (see page 216 of [8] or [7]), and hence we can restrict ourselves to the category of simplicial complexes.

\subsection{Double Complexes}

In this section we introduce the basic notions of a double complex of vector spaces and associated spectral sequences.

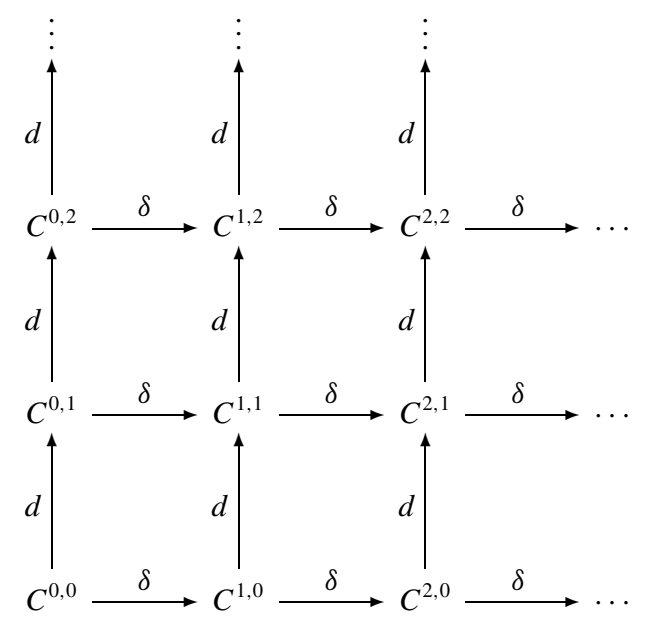


A double complex is a bi-graded vector space,

$$
C=\oplus C^{p, q},
$$

with co-boundary operators $d: C^{p, q} \rightarrow C^{p, q+1}$ and $\delta: C^{p, q} \rightarrow C^{p+1, q}$ and such that $d \delta+\delta d=0$. In our case the double complex would be a single quadrant double complex, which means that we can assume that $C^{p, q}=0$ if either $p<0$ or $q<0$.

Out of a double complex we can form an ordinary complex of vector spaces, namely, the associated total complex, which is a graded vector space, defined by $C^{n}=\bigoplus_{p+q=n}$ $C^{p, q}$, with co-boundary operator $D=d+\delta: C^{n} \rightarrow C^{n+1}$.



There is a natural decreasing filtration that we can define on the associated total complex, by restricting $p$ to be greater than or equal to $k$. We call this the vertical filtration. Similarly, by restricting $q$ to be greater than or equal to some $k$, we obtain a horizontal filtration. These two filtrations play an important role in the next section.

Now consider the vertical filtration.

We denote by $C_{k}^{n}$ the $n$th graded piece of this complex. In other words,

$$
C_{k}^{n}=\bigoplus_{p+q=n, p \geq k} C^{p, q} .
$$

We denote

$$
\begin{gathered}
Z_{k}^{n}=\left\{z \in C_{k}^{n} \mid D z=0\right\}, \\
B^{n}=D C_{n-1}
\end{gathered}
$$

and

$$
H_{k}^{n}=Z_{k}^{n} / Z_{k}^{n} \cap B^{n}
$$


We thus have a decreasing filtration, $\cdots \supset H_{k-1}^{n} \supset H_{k}^{n} \supset H_{k+1}^{n} \cdots$, of the cohomology group $H_{D}^{n}(C)$. We denote the successive quotients $H_{k}^{n} / H_{k+1}^{n}$ by $H^{k, n-k}$.

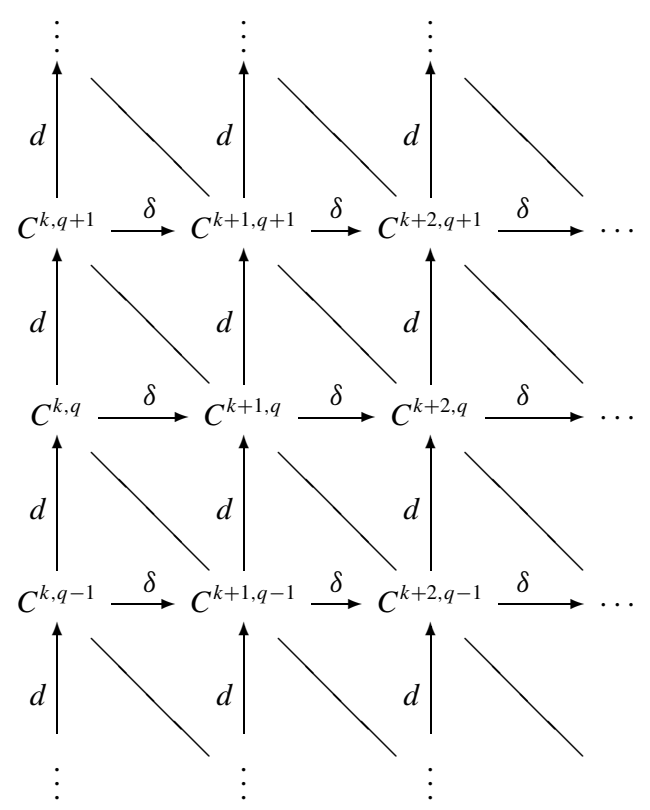

The Leray spectral sequence is a sequence of complexes $\left(E_{r}, d_{r}\right)$ such that $E_{r+1}=$ $H_{d_{r}}\left(E_{r}\right)$.

Any element in $C^{n}=\bigoplus_{i+j=n} C^{i, j}$ will have a leading term at a position $(p, q)$, where $p$ denotes the smallest $i$ such that the component at position $(i, n-i)$ does not vanish.

Let $Z^{p, q}$ denote the set of the $(p, q)$ components of co-cycles whose leading term is at position $\left(p^{\prime}, q^{\prime}\right)$, with $p^{\prime} \geq p$ and $p^{\prime}+q^{\prime}=p+q$. In other words, $Z^{p, q}$ denotes the set of all $a \in C^{p, q}$ such that the following system of equations has a solution:

$$
\begin{aligned}
d a & =0, \\
\delta a & =-d a^{(1)}, \\
\delta a^{(1)} & =-d a^{(2)}, \\
\delta a^{(2)} & =-d a^{(3)}
\end{aligned}
$$

Here, $a^{(i)} \in C^{p+i, q-i}$. Hence, the element $a \oplus a^{(1)} \oplus a^{(2)} \ldots$ lies in $Z_{p}^{p+q}$ with $a \in Z^{p, q}$. 
Similarly, let $B^{p, q} \subset C^{p, q}$ consist of all $b$ with the property that the following system of equations admits a solution:

$$
\begin{aligned}
d b^{(0)}+\delta b^{(-1)} & =b, \\
d b^{(-1)}+\delta b^{(-2)} & =0, \\
d b^{(-2)}+\delta b^{(-3)} & =0
\end{aligned}
$$

Here, $b^{(-i)} \in C^{p-i, q+i-1}$.

It is easy to see that, $H^{p, q} \cong Z^{p, q} / B^{p, q}$.

Now, let

$$
Z_{r}^{p, q}=\left\{a \in C^{p, q} \mid \exists\left(a^{(1)}, \ldots, a^{(r-1)} \mid\left(a, a^{(1)}, \ldots, a^{(r-1)}\right) \text { satisfies (1) }\right\} .\right.
$$

Also, let

$$
\begin{gathered}
B_{r}^{p, q}=\left\{b \in C^{p, q}\left|\exists\left(b^{(0)}, b^{(-1)}, \ldots\right)\right|\left(b, b^{(0)}, b^{(-1)}, \ldots\right)\right. \\
\text { satisfies (2) with } \left.b^{-r}=b^{-r+1}=\cdots=0\right\} .
\end{gathered}
$$

We thus have a sequence of vector subpaces of $C^{p, q}$ satisfying

$$
B_{1}^{p, q} \subset B_{2}^{p, q} \subset \cdots \subset B^{p, q} \subset Z^{p, q} \subset Z_{1}^{p, q} \subset \cdots \subset C^{p, q} .
$$

The $(p, q)$ th graded piece, $E_{r}^{p, q}$, of the $r$ th element, $E_{r}$, of the spectral sequence is defined by $E_{r}^{p, q}=Z_{r}^{p, q} / B_{r}^{p, q}$. It should be seen as an approximation to $H^{p, q}=$ $Z^{p, q} / B^{p, q}$.

We will now define the differentials $d_{r}$. Let $[a] \in E_{r}^{p, q}$ for some $a \in Z_{r}^{p, q}$. Then there exists $a^{(1)}, \ldots, a^{(r-1)}$ satisfying (1). It is a fact (see [13]) that the homomorphism, $d_{r}: E_{r}^{p, q} \rightarrow E_{r}^{p+r, q-r+1}$ (Fig. 2), defined by

$$
d_{r}[a]=\left[\delta a^{(r-1)}\right] \in E_{r}^{p+r, q-r+1},
$$

is well defined (that is, independent of the choice of the representative $a$ ).

The sequence of graded complexes, $\left(E_{r}, d_{r}\right)$, where the complex $E_{r+1}$ is obtained from $E_{r}$ by taking its homology with respect to $d_{r}$ (that is, $E_{r+1}=H_{d_{r}}\left(E_{r}\right)$ ) is called the spectral sequence associated to the double complex $C^{p, q}$.

\subsection{The Mayer-Vietoris Double Complex}

In this section we describe the double complex of interest to us-namely, the one arising from the Mayer-Vietoris exact sequence.

Let $A_{1}, \ldots, A_{n}$ be sub-complexes of a finite simplicial complex $A$ such that $A=$ $A_{1} \cup \cdots \cup A_{n}$. Note that the intersections of any number of the sub-complexes, $A_{i}$, is again a sub-complex of $A$. We denote by $A_{\alpha_{0}, \ldots, \alpha_{p}}$ the sub-complex $A_{\alpha_{0}} \cap \cdots \cap A_{\alpha_{p}}$. 


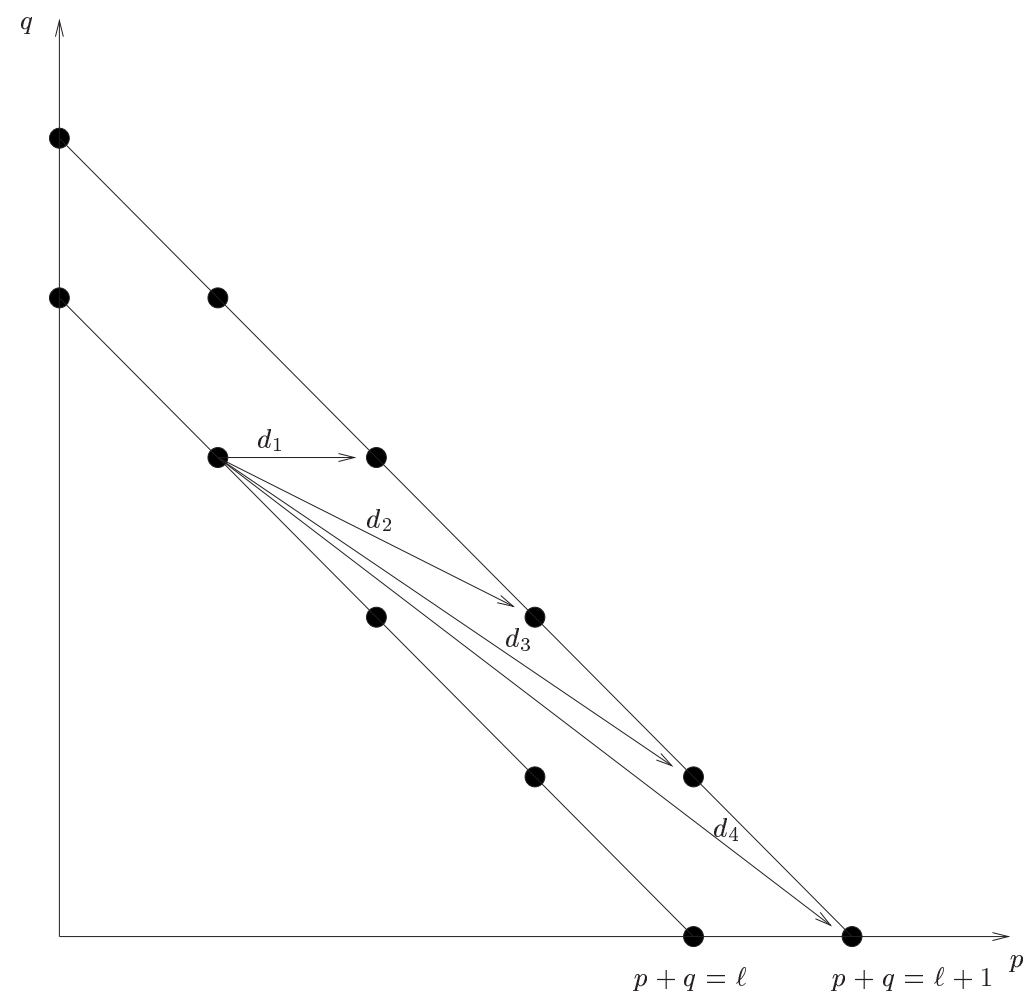

Fig. 2. $d_{r}: E_{r}^{p, q} \rightarrow E_{r}^{p+r, q-r+1}$.

Let $C^{i}(A)$ denote the $\mathbb{R}$-vector space of $i$ co-chains of $A$, and $C^{*}(A)=\oplus_{i} C^{i}(A)$. We will denote by $d: C^{q}(A) \rightarrow C^{q+1}(A)$ the usual co-boundary homomorphism. More precisely, given $\omega \in C^{q}(A)$, and a $q+1$ simplex $\left[a_{0}, \ldots, a_{q+1}\right] \in A$,

$$
d \omega\left(\left[a_{0}, \ldots, a_{q+1}\right]\right)=\sum_{0 \leq i \leq q+1}(-1)^{i} \omega\left(\left[a_{0}, \ldots, \hat{a}_{i}, \ldots, a_{q+1}\right]\right)
$$

(here and everywhere else in the paper ${ }^{\wedge}$ denotes omission). Now extend $d \omega$ to a linear form on all of $C_{q+1}(A)$ by linearity, to obtain an element of $C^{q+1}(A)$.

Recall that a sequence of vector space homomorphisms

$$
\cdots \stackrel{d_{i-1}}{\longrightarrow} V_{i} \stackrel{d_{i}}{\longrightarrow} V_{i+1} \stackrel{d_{i+1}}{\longrightarrow} \cdots
$$

is said to be exact if $\operatorname{ker}\left(d_{i}\right)=\operatorname{Im}\left(d_{i-1}\right)$ for each $i$.

The Mayer-Vietoris exact sequence is an exact sequence of vector spaces, each of the form $\Pi_{\alpha_{0}<\cdots<\alpha_{p}} C^{*}\left(A_{\alpha_{0}, \ldots, \alpha_{p}}\right)$. (Here and everywhere else in the paper $\Pi$ denotes the direct product of vector spaces.) The connecting homomorphisms are "generalized" restrictions and will be defined below. 
The Mayer-Vietoris exact sequence is the following:

$$
\begin{aligned}
& 0 \longrightarrow C^{*}(A) \stackrel{r}{\longrightarrow} \Pi_{\alpha_{0}} C^{*}\left(A_{\alpha_{0}}\right) \stackrel{\delta}{\longrightarrow} \Pi_{\alpha_{0}<\alpha_{1}} C^{*}\left(A_{\alpha_{0}, \alpha_{1}}\right) \cdots \\
& \quad \stackrel{\delta}{\longrightarrow} \Pi_{\alpha_{0}<\cdots<\alpha_{p}} C^{*}\left(A_{\alpha_{0}, \ldots, \alpha_{p}}\right) \cdots \stackrel{\delta}{\longrightarrow} \Pi_{\alpha_{0}<\cdots<\alpha_{p+1}} C^{*}\left(A_{\alpha_{0}, \ldots, \alpha_{p+1}}\right) \cdots \stackrel{\delta}{\longrightarrow} \cdots,
\end{aligned}
$$

where $r$ is induced by restriction and the connecting homomorphisms $\delta$ are described below.

Given an $\omega \in \Pi_{\alpha_{0}<\cdots<\alpha_{p}} C^{q}\left(A_{\alpha_{0}, \ldots, \alpha_{p}}\right)$ we define $\delta(\omega)$ as follows: First note that $\delta(\omega) \in \Pi_{\alpha_{0}<\cdots<\alpha_{p+1}} C^{q}\left(A_{\alpha_{0}, \ldots, \alpha_{p+1}}\right)$, and it suffices to define $\delta(\omega)_{\alpha_{0}, \ldots, \alpha_{p+1}}$ for each $(p+2)$ tuple $0 \leq \alpha_{0}<\cdots<\alpha_{p+1} \leq n$. Note that $\delta(\omega)_{\alpha_{0}, \ldots, \alpha_{p+1}}$ is a linear form on the vector space, $C_{q}\left(A_{\alpha_{0}, \ldots, \alpha_{p+1}}\right)$, and hence is determined by its values on the $q$-simplices in the complex $A_{\alpha_{0}, \ldots, \alpha_{p+1}}$. Furthermore, each $q$-simplex, $s \in A_{\alpha_{0}, \ldots, \alpha_{p+1}}$, is automatically a simplex of the complexes $A_{\alpha_{0}, \ldots, \hat{\alpha}_{i}, \ldots, \alpha_{p+1}}, 0 \leq i \leq p+1$.

We define

$$
(\delta \omega)_{\alpha_{0}, \ldots, \alpha_{p+1}}(s)=\sum_{0 \leq i \leq p+1}(-1)^{i} \omega_{\alpha_{0}, \ldots, \hat{\alpha}_{i}, \ldots, \alpha_{p+1}}(s) .
$$

Lemma 1. The sequence defined above is exact.

Proof. We first prove that $\delta^{2}=0$. For $\omega \in \Pi_{\alpha_{0}<\cdots<\alpha_{p}} C^{*}\left(A_{\alpha_{0}, \ldots, \alpha_{p}}\right)$,

$$
\begin{aligned}
\delta^{2}(\omega)_{\alpha_{0}, \ldots, \alpha_{p+2}}= & \sum_{0 \leq i \leq p+2}(-1)^{i}(\delta \omega)_{\alpha_{0}, \ldots, \hat{\alpha_{i}} \cdots \alpha_{p+2}} \\
= & \sum_{i, j<i}(-1)^{i}(-1)^{j} \omega_{\alpha_{0}, \ldots, \hat{\alpha_{j}}, \ldots, \hat{\alpha}_{i}, \ldots, \alpha_{p+2}} \\
& +\sum_{i, j>i}(-1)^{i}(-1)^{j} \omega_{\alpha_{0}, \ldots, \hat{\alpha_{i}}, \ldots, \hat{\alpha_{j} \cdots \alpha_{p+2}}}=0 .
\end{aligned}
$$

Now let $\omega \in \Pi_{\alpha_{0}<\cdots<\alpha_{p}} C^{*}\left(A_{\alpha_{0}, \ldots, \alpha_{p}}\right)$ such that $\delta \omega=0$. For a simplex $s \in A$ let $n(s)=$ $\#\left\{A_{\alpha} \mid s \in A_{\alpha}\right\}$.

Define $\tau \in \Pi_{\alpha_{0}<\cdots<\alpha_{p-1}} C^{*}\left(A_{\alpha_{0}, \ldots, \alpha_{p-1}}\right)$ by

$$
\tau_{\alpha_{0}, \ldots, \alpha_{p-1}}(s)=\frac{1}{n(s)} \sum_{\alpha \mid s \in A_{\alpha}} \omega_{\alpha, \alpha_{0}, \ldots, \alpha_{p-1}}(s) .
$$

Now,

$$
\begin{aligned}
(\delta \tau)_{\alpha_{0}, \ldots, \alpha_{p}}(s) & =\sum_{0 \leq i \leq p}(-1)^{i} \tau_{\alpha_{0}, \ldots, \hat{\alpha_{i}}, \ldots, \alpha_{p}}(s) \\
& =\sum_{0 \leq i \leq p}(-1)^{i}\left(\frac{1}{n(s)} \sum_{\alpha \mid s \in A_{\alpha}} \omega_{\alpha, \alpha_{0}, \ldots, \hat{\alpha}_{i}, \ldots, \alpha_{p}}(s)\right) .
\end{aligned}
$$


Now $\delta \omega=0$. Hence,

$$
(\delta \omega)_{\alpha, \alpha_{0}, \ldots, \alpha_{p}}(s)=\omega_{\alpha_{0}, \ldots, \alpha_{p}}(s)-\sum_{0 \leq i \leq p}(-1)^{i+1} \omega_{\alpha, \alpha_{0}, \ldots, \hat{\alpha}_{i}, \ldots, \alpha_{p}}(s)=0 .
$$

Hence,

$$
\omega_{\alpha_{0}, \ldots, \alpha_{p}}(s)=\sum_{0 \leq i \leq p}(-1)^{i} \omega_{\alpha, \alpha_{0}, \ldots, \hat{\alpha}_{i}, \ldots, \alpha_{p}}(s) .
$$

Substituting the above in the expression for $\delta \tau$ above, we have

$$
\begin{aligned}
(\delta \tau)_{\alpha_{0}, \ldots, \alpha_{p}}(s) & =\frac{1}{n(s)} \sum_{\alpha \mid s \in A_{\alpha}}\left(\sum_{0 \leq i \leq p}(-1)^{i} \omega_{\alpha, \alpha_{0}, \ldots, \hat{\alpha}_{i}, \ldots, \alpha_{p}}(s)\right) \\
& =\frac{1}{n(s)} \sum_{\alpha \mid s \in A_{\alpha}} \omega_{\alpha_{0}, \ldots, \alpha_{p}}(s)=\omega_{\alpha_{0}, \ldots, \alpha_{p}}(s) .
\end{aligned}
$$

This proves that the sequence is exact.

We now consider the following bi-graded double complex $\mathcal{M}^{p, q}$, with a total differential $D=\delta+(-1)^{p} d$, where

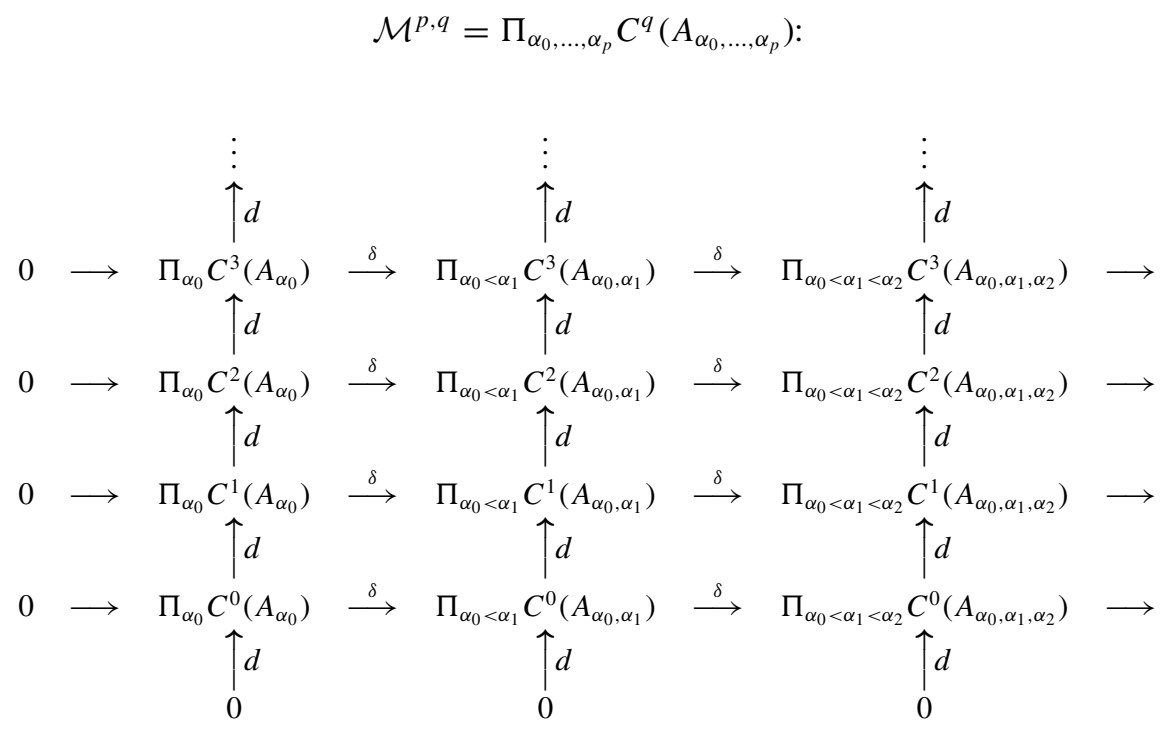

There are two spectral sequences (corresponding to taking horizontal or vertical filtrations, respectively) associated with $\mathcal{M}^{p, q}$ both converging to $H_{D}^{*}(\mathcal{M})$. The first terms of these are $E_{1}=H_{\delta} \mathcal{M}, E_{2}=H_{d} H_{\delta} \mathcal{M}$ and $E_{1}^{\prime}=H_{d} \mathcal{M}, E_{2}^{\prime}=H_{\delta} H_{d} \mathcal{M}$. Because of 
the exactness of the generalized Mayer-Vietoris sequence, we have that

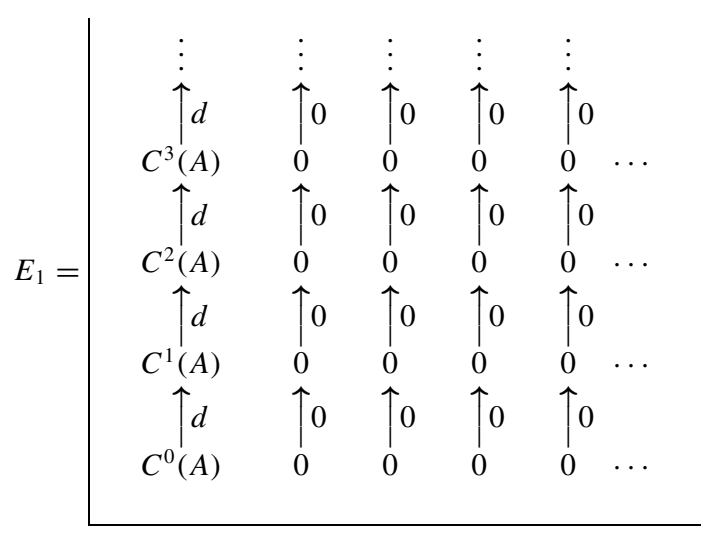

and

$$
E_{2}=\mid \begin{array}{ccccccc}
\vdots & \vdots & \vdots & \vdots & \vdots & \vdots & \\
H^{3}(A) & 0 & 0 & 0 & 0 & 0 & \cdots \\
H^{2}(A) & 0 & 0 & 0 & 0 & 0 & \cdots \\
H^{1}(A) & 0 & 0 & 0 & 0 & 0 & \cdots \\
H^{0}(A) & 0 & 0 & 0 & 0 & 0 & \cdots
\end{array}
$$

The degeneration of this sequence at $E_{2}$ shows that $H_{D}^{*}(\mathcal{M}) \cong H^{*}(A)$.

The initial term $E_{1}^{\prime}$ of the second spectral sequence is given by

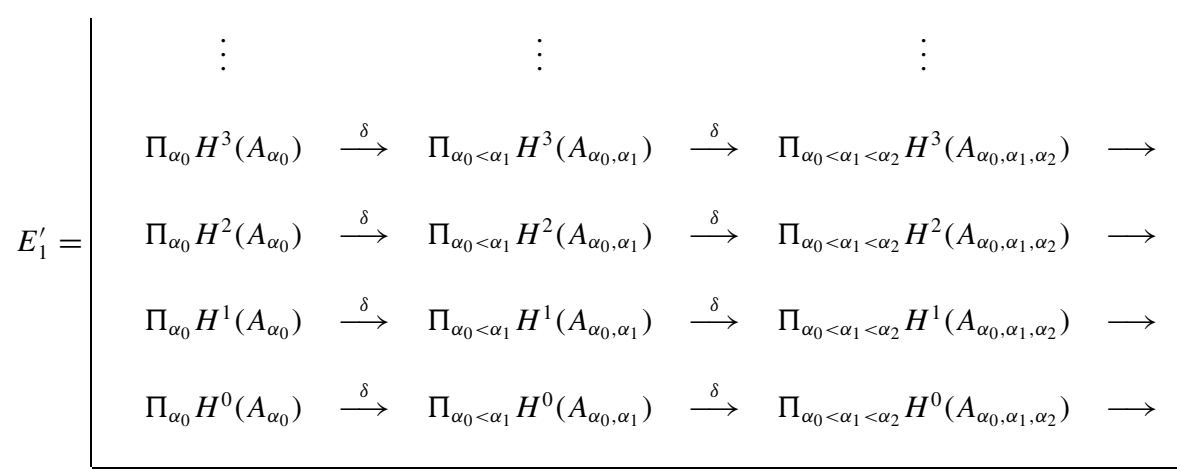


Since this spectral sequence also converges to $H^{*}(A)$, we have that

$$
\operatorname{rank} H^{i}(A) \leq \sum_{p+q=i} \operatorname{rank} E_{1}^{\prime p, q}
$$

The following lemma is an easy consequence.

Lemma 2. Let $A$ be a finite simplicial complex and let $A_{1}, \ldots, A_{n}$ be sub-complexes of $A$ such that $A=A_{1} \cup \cdots \cup A_{n}$. Then for every $i \geq 0$,

$$
b_{i}(A) \leq \sum_{j=1}^{i+1} \sum_{J \subset\{1, \ldots, n\}, \#(J)=j} b_{i-j+1}\left(A_{J}\right) .
$$

Proof. From the discussion above,

$$
\operatorname{rank} E_{1}^{\prime \ell, i-\ell} \leq \sum_{J \subset\{1, \ldots, n\}, \#(J)=\ell+1} b_{i-\ell}\left(A_{J}\right)
$$

The inequality follows.

Now let $A_{1}, \ldots, A_{n}$ be sub-complexes of a finite simplicial complex of dimension $k^{\prime}$, and let $A=A_{1} \cap \cdots \cap A_{n}$. Let $A^{\alpha_{0}, \ldots, \alpha_{p}}$ denote the union, $A_{\alpha_{0}} \cup \cdots \cup A_{\alpha_{p}}$. Let $C_{i}(A)$ denote the $\mathbb{R}$-vector space of $i$ chains of $A$, and $C_{*}(A)=\bigoplus_{i} C_{i}(A)$.

Consider the following sequence of homomorphisms:

$$
\begin{aligned}
& 0 \longrightarrow C_{*}(A) \stackrel{i}{\longrightarrow} \Pi_{\alpha_{0}} C_{*}\left(A^{\alpha_{0}}\right) \stackrel{\delta}{\longrightarrow} \Pi_{\alpha_{0}<\alpha_{1}} C_{*}\left(A^{\alpha_{0}, \alpha_{1}}\right) \cdots \\
& \quad \stackrel{\delta}{\longrightarrow} \Pi_{\alpha_{0}<\cdots<\alpha_{p}} C_{*}\left(A^{\alpha_{0}, \ldots, \alpha_{p}}\right) \cdots \stackrel{\delta}{\longrightarrow} \prod_{\alpha_{0}<\cdots<\alpha_{p+1}} C_{*}\left(A^{\alpha_{0}, \ldots, \alpha_{p+1}}\right) \cdots \stackrel{\delta}{\longrightarrow} \cdots,
\end{aligned}
$$

where $i$ is induced by inclusion and the connecting homomorphisms $\delta$ are defined as follows:

for $c \in \Pi_{\alpha_{0}<\cdots<\alpha_{p}} C_{*}\left(A^{\alpha_{0}, \ldots, \alpha_{p}}\right)$, we let

$$
(\delta c)_{\alpha_{0}, \ldots, \alpha_{p+1}}=\sum_{0 \leq i \leq p+1}(-1)^{i} c_{\alpha_{0}, \ldots, \hat{\alpha_{i}}, \ldots, \alpha_{p+1}} .
$$

Lemma 3. The sequence defined above is exact.

Proof. The proof is similar to that of Lemma 1. 
We have a corresponding bi-graded double complex $\mathcal{N}_{p, q}$ with total differential $D=$ $\delta+(-1)^{p} \partial$, with

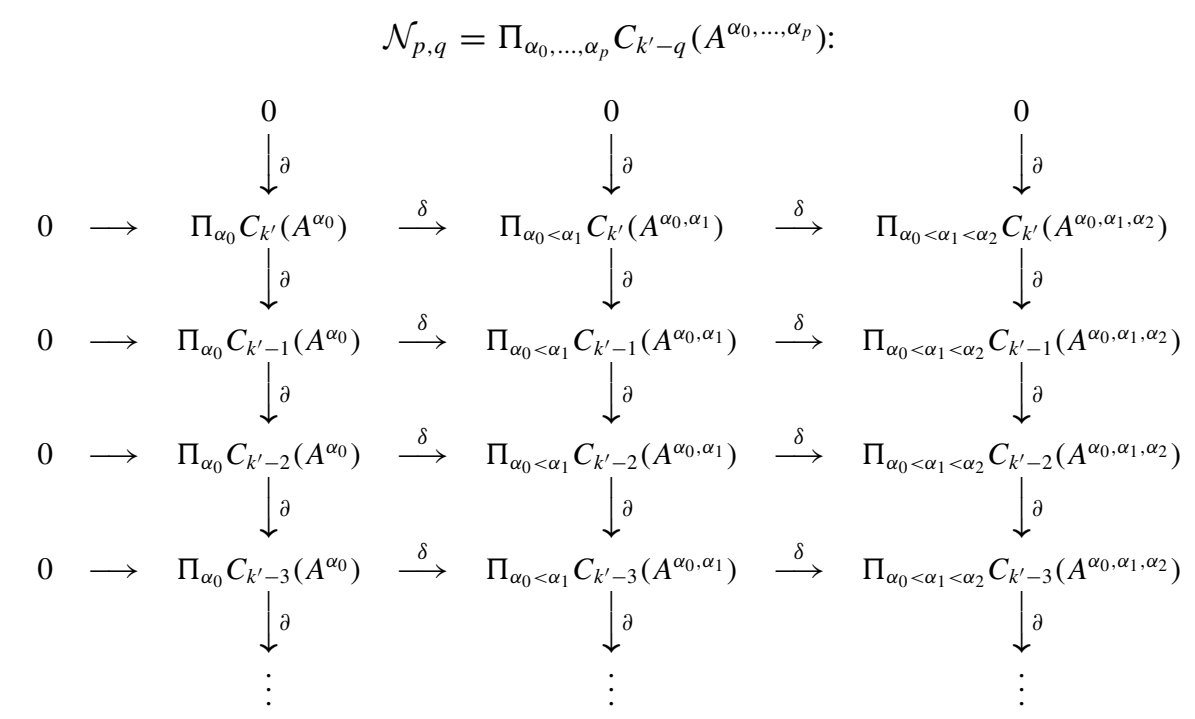

As in the proof of Lemma 2 we obtain two spectral sequences, $E, E^{\prime}$.

Here

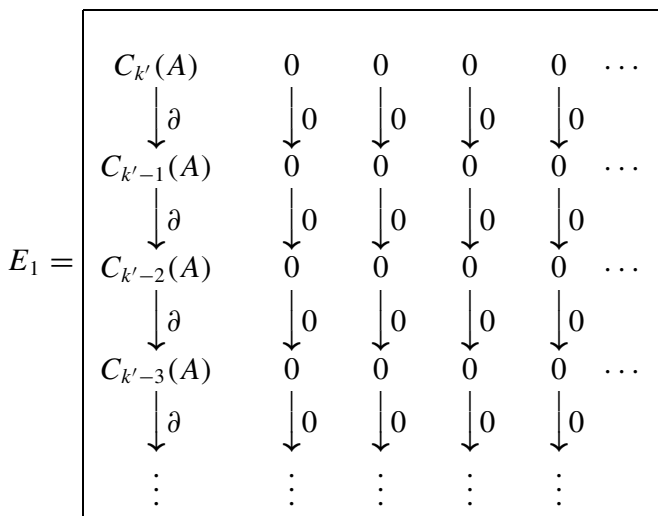

and

$$
E_{2}=\begin{array}{cccccc}
\hline H_{k^{\prime}}(A) & 0 & 0 & 0 & 0 & \cdots \\
H_{k^{\prime}-1}(A) & 0 & 0 & 0 & 0 & \cdots \\
H_{k^{\prime}-2}(A) & 0 & 0 & 0 & 0 & \cdots \\
H_{k^{\prime}-3}(A) & 0 & 0 & 0 & 0 & \cdots \\
\vdots & \vdots & \vdots & \vdots & \vdots &
\end{array}
$$


Similarly,

$$
E_{1}^{\prime}=\left(\begin{array}{ccccc}
\Pi_{\alpha_{0}} H_{k^{\prime}}\left(A^{\alpha_{0}}\right) & \stackrel{\delta}{\longrightarrow} & \Pi_{\alpha_{0}<\alpha_{1}} H_{k^{\prime}}\left(A^{\alpha_{0}, \alpha_{1}}\right) & \stackrel{\delta}{\longrightarrow} & \Pi_{\alpha_{0}<\alpha_{1}<\alpha_{2}} H_{k^{\prime}}\left(A^{\alpha_{0}, \alpha_{1}, \alpha_{2}}\right) \\
\Pi_{\alpha_{0}} H_{k^{\prime}-1}\left(A^{\alpha_{0}}\right) & \stackrel{\delta}{\longrightarrow} & \Pi_{\alpha_{0}<\alpha_{1}} H_{k^{\prime}-1}\left(A^{\alpha_{0}, \alpha_{1}}\right) & \stackrel{\delta}{\longrightarrow} & \Pi_{\alpha_{0}<\alpha_{1}<\alpha_{2}} H_{k^{\prime}-1}\left(A^{\alpha_{0}, \alpha_{1}, \alpha_{2}}\right) \\
\Pi_{\alpha_{0}} H_{k^{\prime}-2}\left(A^{\alpha_{0}}\right) & \stackrel{\delta}{\longrightarrow} & \Pi_{\alpha_{0}<\alpha_{1}} H_{k^{\prime}-2}\left(A^{\alpha_{0}, \alpha_{1}}\right) & \stackrel{\delta}{\longrightarrow} & \Pi_{\alpha_{0}<\alpha_{1}<\alpha_{2}} H_{k^{\prime}-2}\left(A^{\alpha_{0}, \alpha_{1}, \alpha_{2}}\right) \\
\Pi_{\alpha_{0}} H_{k^{\prime}-3}\left(A^{\alpha_{0}}\right) & \stackrel{\delta}{\longrightarrow} & \Pi_{\alpha_{0}<\alpha_{1}} H_{k^{\prime}-3}\left(A^{\alpha_{0}, \alpha_{1}}\right) & \stackrel{\delta}{\longrightarrow} & \Pi_{\alpha_{0}<\alpha_{1}<\alpha_{2}} H_{k^{\prime}-3}\left(A^{\alpha_{0}, \alpha_{1}, \alpha_{2}}\right) \\
\vdots & & \vdots & & \\
& & & & \vdots
\end{array}\right.
$$

Lemma 4. Let $A_{1}, \ldots, A_{n}$ be sub-complexes of a finite simplicial complex $T$ of dimension $k^{\prime}$, and let $A=A_{1} \cap \cdots \cap A_{n}$. Then, for every $0 \leq i \leq k^{\prime}$,

$$
\begin{aligned}
b_{i}(A) \leq & \sum_{j=1}^{k^{\prime}-i-1} \sum_{J \subset\{1, \ldots, n\}, \#(J)=j} b_{i+j-1}\left(A^{J}\right) \\
& +\sum_{J \subset\{1, \ldots, n\}, \#(J)=k^{\prime}-i}\left(b_{k^{\prime}-1}\left(A^{J}\right)+b_{k^{\prime}}(T)\right) .
\end{aligned}
$$

Proof. The proof is similar to that of the proof of Lemma 2 using instead the two spectral sequences associated to $\mathcal{N}$ whose initial terms are given above. Unlike in the proof of Lemma 2, in the case when $k^{\prime}<k$, we need to refine our estimate by looking at certain terms of $E_{2}^{\prime}$.

From the spectral sequence $E^{\prime}$ we obtain that

$$
b_{i}(A) \leq \sum_{j=0}^{k^{\prime}-i} \operatorname{rank}\left(E_{1}^{\prime j, i+j}\right) \leq \sum_{j=0}^{k^{\prime}-i-1} \operatorname{rank}\left(E_{1}^{\prime j, i+j}\right)+\operatorname{rank}\left(E_{2}^{\prime k^{\prime}-i, k^{\prime}}\right)
$$

From $E_{1}^{\prime}$ we get that $\operatorname{rank}\left(E_{1}^{\prime j, i+j}\right) \leq \sum_{J \subset\{1, \ldots, n\}, \#(J)=j+1} b_{i+j}\left(A^{J}\right)$.

In order to finish the proof we have to bound $\operatorname{rank}\left(E_{2}^{\prime k^{\prime}-i+1, k^{\prime}}\right)$. Notice that the top row, $E_{2}^{\prime p, k^{\prime}}$, is obtained by taking the homology of the top row of $E_{1}^{\prime}$ with respect to $\delta$. Now in the highest dimension, $k^{\prime}$, the inclusion homomorphisms $i_{*}: H_{k^{\prime}}\left(A^{J}\right) \rightarrow H_{k^{\prime}}(T)$ are all injective. Hence, each $H_{k^{\prime}}\left(A^{J}\right)$ can be identified with a certain subspace of $H_{k^{\prime}}(T)$.

Let $V_{J}=i_{*}\left(H_{k^{\prime}}\left(A^{J}\right)\right)$ and $V=H_{k^{\prime}}(T)$ and consider the homomorphism,

$$
\delta_{p}: \Pi_{\alpha_{0}<\cdots<\alpha_{p}} V_{\alpha_{0}, \ldots, \alpha_{p}} \rightarrow \Pi_{\alpha_{0}<\cdots<\alpha_{p+1}} V_{\alpha_{0}, \ldots, \alpha_{p+1}}
$$

given by

$$
\left(\delta_{p} v\right)_{\alpha_{0}, \ldots, \alpha_{p+1}}=\sum_{0 \leq j \leq p+1}(-1)^{j} v_{\alpha_{0}, \ldots, \hat{\alpha}_{j}, \ldots, \alpha_{p+1}},
$$


for $v \in \Pi_{\alpha_{0}<\cdots<\alpha_{p}} V_{\alpha_{0}, \ldots, \alpha_{p}}$. It follows that for $v \in \operatorname{ker}\left(\delta_{p}\right), v_{1, \alpha_{1}, \ldots, \alpha_{p}}, 1<\alpha_{1}<\cdots<$ $\alpha_{p} \leq n$, determines $v$. Hence, $\operatorname{rank}\left(E_{2}^{\prime p, k^{\prime}}\right) \leq\left(\begin{array}{l}n \\ p\end{array}\right) \operatorname{rank}\left(H_{k^{\prime}}(T)\right)$.

Hence, $\operatorname{rank}\left(E_{2}^{\prime k^{\prime}-i, k^{\prime}}\right) \leq\left(\begin{array}{c}n \\ k^{\prime}-i\end{array}\right) b_{k^{\prime}}(T)$. The lemma now follows from the inequalities proved above.

\section{Proofs of the Main Results}

In order to apply Lemmas 2 and 4 in our situation, we will need a bound on the sum of the Betti numbers of sets defined by the disjunction (respectively conjunction) of a small number of inequalities. We proceed to prove these bounds.

We first recall the classical Mayer-Vietoris sequence for cohomologies. Let $A, B \subset$ $R^{k}$ be closed and bounded semi-algebraic sets. Then the Mayer-Vietoris sequence is the following exact sequence of cohomology groups [17]:

$$
\begin{aligned}
0 & \leftarrow H^{k-1}(A \cap B) \leftarrow H^{k-1}(A) \oplus H^{k-1}(B) \leftarrow H^{k-1}(A \cup B) \\
& \leftarrow H^{k-2}(A \cap B) \leftarrow \cdots \leftarrow H^{i+1}(A \cup B) \leftarrow H^{i}(A \cap B) \\
& \leftarrow H^{i}(A) \oplus H(B) \leftarrow H^{i}(A \cup B) \leftarrow \cdots .
\end{aligned}
$$

The following lemma is an easy consequence.

Lemma 5. Let $S_{1}, S_{2}$ be two closed and bounded semi-algebraic sets. Then

$$
\begin{aligned}
b_{i}\left(S_{1}\right)+b_{i}\left(S_{2}\right) & \leq b_{i}\left(S_{1} \cup S_{2}\right)+b_{i}\left(S_{1} \cap S_{2}\right), \\
b_{i}\left(S_{1} \cup S_{2}\right) & \leq b_{i}\left(S_{1}\right)+b_{i}\left(S_{2}\right)+b_{i-1}\left(S_{1} \cap S_{2}\right), \\
b_{i}\left(S_{1} \cap S_{2}\right) & \leq b_{i}\left(S_{1}\right)+b_{i}\left(S_{2}\right)+b_{i+1}\left(S_{1} \cup S_{2}\right)
\end{aligned}
$$

We also recall the Oleinik/Petrovskii/Thom/Milnor bound on the sum of the Betti numbers of algebraic sets. Let $b(k, d)$ be the maximum of the sum of the Betti numbers of any algebraic set defined by polynomials of degree $d$ in $R^{k}$.

The Oleinik/Petrovskii/Thom/Milnor [15], [19], [14] bound is the following:

$$
b(k, d) \leq d(2 d-1)^{k-1} .
$$

For the rest of the paper, we fix a closed ball $B(0, r)$ with center 0 and radius $r$ big enough so that, for every semi-algebraic set $S$ that we consider (defined by Boolean formulas involving sign conditions on a fixed family of $\left.\left\{Q, P_{1}, \ldots, P_{n}\right\}\right), H_{*}(S)$ and $H_{*}(S \cap B(0, r))$ are isomorphic. This is always possible by the local conical structure at infinity of semi-algebraic sets [8, page 225]. Finally, we define the sets $Z$ and $Z_{r}$ by

$$
Z=Z(Q), \quad Z_{r}=Z \cap B(0, r)
$$

We have the following lemma bounding the Betti numbers of sets defined by the disjunction of few inequalities. 
Lemma 6. Let $R$ be a real closed field and let $V_{1} \subset R^{k}$ be the set defined by the disjunction of $\ell$ inequalities,

$$
\begin{aligned}
P_{1} \geq 0, \ldots, P_{\ell} \geq 0, & P_{i} \in R\left[X_{1}, \ldots, X_{k}\right], \\
\operatorname{deg}\left(P_{i}\right) \leq d, & 1 \leq i \leq \ell,
\end{aligned}
$$

contained in a variety $Z(Q)$ of real dimension $k^{\prime}$ with $\operatorname{deg}(Q) \leq d$. Then, for all $i, 0 \leq i \leq k^{\prime}$,

$$
b_{i}\left(V_{1}\right) \leq 2^{\ell} d(2 d-1)^{k-1} .
$$

Proof. Let

$$
\begin{aligned}
W_{0} & =\bigcup_{1 \leq i \leq \ell} Z\left(P_{i}\right) \cap Z_{r}, \\
W_{1} & =\left\{x \in R^{k} \mid \bigvee_{1 \leq i \leq \ell} P_{i}(x) \geq 0\right\} \cap Z_{r}, \\
F & =\left\{x \in R^{k} \mid\left(\bigwedge_{1 \leq i \leq \ell} P_{i}(x) \leq 0\right) \vee\left(\bigvee_{1 \leq i \leq \ell} P_{i}(x)=0\right)\right\} \cap Z_{r} .
\end{aligned}
$$

Lemma 7. For all $i, 0 \leq i \leq k^{\prime}$,

$$
b_{i}\left(W_{0}\right) \leq\left(2^{\ell}-1\right) d(2 d-1)^{k-1} .
$$

Proof. Using Lemma 2 observe that $b_{i}\left(W_{0}\right)$ can be bounded by the sum of the Betti numbers of all sets that can be obtained by taking $j$-ary intersections, for $1 \leq j \leq \ell$, of the sets $Z\left(P_{i}\right) \cap Z_{r}$.

The number of possible $j$-ary intersections is $\left(\begin{array}{l}\ell \\ j\end{array}\right)$.

The sum of the Betti numbers of each of these sets is bounded by $d(2 d-1)^{k-1}$ by the Oleinik-Petrovskii/Thom/Milnor bound (8). Thus,

$$
b_{i}\left(W_{0}\right) \leq \sum_{j=1}^{\ell}\left(\begin{array}{l}
\ell \\
j
\end{array}\right) d(2 d-1)^{k-1}=\left(2^{\ell}-1\right) d(2 d-1)^{k-1} .
$$

Lemma 8. For all $i, 0 \leq i \leq k^{\prime}$,

$$
b_{i}\left(W_{1}\right) \leq 2^{\ell} d(2 d-1)^{k-1} .
$$

Proof. Note that

$$
W_{1} \cup F=Z_{r}
$$

and

$$
W_{1} \cap F=W_{0} .
$$


Now apply inequality (5) to get

$$
b_{i}\left(W_{1}\right) \leq b_{i}\left(W_{1} \cap F\right)+b_{i}\left(W_{1} \cup F\right)=b_{i}\left(W_{0}\right)+b_{i}\left(Z_{r}\right) .
$$

We conclude using Lemma 7.

Finally, Lemma 6 follows from Lemma 8 since $H_{*}\left(V_{1}\right) \cong H_{*}\left(W_{1}\right)$ by our choice of $r$.

Lemma 9. Let $R$ be a real closed field and let $V_{2} \subset R^{k}$ be the set defined by the conjunction of $\ell$ inequalities,

$$
\begin{aligned}
P_{1} \geq 0, \ldots, P_{\ell} \geq 0, & P_{i} \in R\left[X_{1}, \ldots, X_{k}\right], \\
\operatorname{deg}\left(P_{i}\right) \leq d, & 1 \leq i \leq \ell,
\end{aligned}
$$

contained in a variety $Z(Q)$ of real dimension $k^{\prime}$ with $\operatorname{deg}(Q) \leq d$. Then, for all $i, 0 \leq i \leq k^{\prime}$,

$$
b_{i}\left(V_{2}\right) \leq\left(3^{\ell}-1\right) d(2 d-1)^{k-1} .
$$

Proof. Using Lemma 4 we have that $b_{i}\left(V_{2}\right)$ is bounded by the sum of the $(i+j-1)$ th Betti numbers of all possible $j$-ary unions $1 \leq j \leq \ell$, of the sets $\left\{x \in R^{k} \mid P_{i} \geq 0\right\} \cap Z_{r}$. Using Lemma 6 we get that

$$
b_{i}\left(V_{2}\right) \leq \sum_{1 \leq j \leq \ell}\left(\begin{array}{l}
\ell \\
j
\end{array}\right) 2^{j} d(2 d-1)^{k-1} \leq\left(3^{\ell}-1\right) d(2 d-1)^{k-1} .
$$

We now prove the main theorems using the tools developed above.

Proof of Theorem 3. Let the set $S_{j}$ be defined by $\left(P_{j} \geq 0\right) \cap Z_{r}$. Each $S_{j}$ is closed and bounded and $H_{*}\left(\bigcap_{1 \leq j \leq n} S_{j}\right) \cong H_{*}(S)$. Consider a triangulation of $\bigcup_{1 \leq j \leq n} S_{j}$ which respects the sets $S_{j}$ and all possible intersections. Let $A_{i}$ be the sub-complex corresponding to the set $S_{i}$ in this triangulation.

Now apply Lemma 4 with the complexes $A_{i}$ defined above, and apply the bounds obtained in Lemma 6 on the summands on the right-hand side.

Proof of Theorem 4. Let the set $S_{j}$ be defined by $P_{j} \geq 0 \cap B(0, r)$. Each $S_{j}$ is closed and bounded and $H_{*}\left(\bigcup_{1 \leq j \leq n} S_{j}\right) \cong H_{*}(S)$.

Again, choose a triangulation of the union of the sets which respects all non-empty intersections. Let $A_{i}$ denote the sub-complex corresponding to the set $S_{i}$. Now apply Lemma 2 with the complexes $A_{j}$ defined above, and apply the bounds obtained in Lemma 9 on the summands on the right-hand side.

Proof of Theorem 6. Using the same technique used in the proof of Lemma 9, we can convert the bound in Theorem 5 into a bound on the sum of the Betti numbers of a set 
defined by a disjunction of quadratic inequalities. Namely,

Theorem 7. Let $S \subset R^{k}$ be defined by the disjunction of the inequalities $P_{1} \geq$ $0, \ldots, P_{\ell} \geq 0$, and let $\operatorname{deg}\left(P_{i}\right) \leq 2,1 \leq i \leq \ell$. Then the sum of the Betti numbers of $S$ is bounded above by $k^{O(\ell)}$.

Now use Lemma 4 and the bound in Theorem 7 on the sum of the Betti numbers of the at most $\ell$-ary unions.

\section{Acknowledgments}

The author thanks Andrei Gabrielov for suggesting the use of a spectral sequence argument. A preliminary version of this paper appeared in the Proceedings of the ACM Symposium on Computational Geometry, 2001, where inductive arguments were used to prove the same results.

\section{References}

1. P. K. Agarwal and M. Sharir, Arrangements and their applications, in Handbook of Computational Geometry, J.R. Sack and J. Urrutia (eds.), Elsevier, Amsterdam, 2000, pp. 49-120.

2. A. I. Barvinok, On the Betti numbers of semialgebraic sets defined by few quadratic inequalities, Mathematische Zeitschrift, 225 (1997), 231-244.

3. S. Basu, On bounding the Betti numbers and computing the Euler characteristics of semi-algebraic sets, Discrete \& Computational Geometry, 22 (1999), 1-18.

4. S. Basu, The combinatorial and topological complexity of a single cell, Discrete \& Computational Geometry, 29(1) (2003), 41-59.

5. S. Basu, Computing Betti numbers of arrangements via spectral sequences, Journal of Computer and System Sciences, to appear.

6. S. Basu, R. Pollack and M.-F. Roy, On the number of cells defined by a family of polynomials on a variety, Mathematika, 43 (1996), 120-126.

7. S. Basu, R. Pollack and M.-F. Roy, Algorithms in Real Algebraic Geometry, Springer-Verlag, New York, to appear.

8. J. Bochnak, M. Coste and M.-F. Roy, Real Algebraic Geometry, Springer-Verlag, New York, 1998.

9. H. Edelsbrunner, The union of balls and its dual shape, Discrete \& Computational Geometry, 13 (1995), 415-440.

10. H. Edelsbrunner, D. Letscher and A. Zomorodian, Topological persistence and simplification, Proceedings of the 41st IEEE Symposium on the Foundations Computer Science, pp. 454-463, 2000.

11. D. Halperin, Arrangements, in Handbook of Discrete and Computational Geometry, J. O'Rourke and J. E. Goodman (eds.), CRC Press, Boca Raton, FL, 1997, pp. 389-412.

12. D. Halperin and M. Sharir, Almost tight upper bounds for the single cell and zone problems in three dimensions, Discrete \& Computational Geometry, 14 (1995), 385-410.

13. J. McCleary, A User's Guide to Spectral Sequences, 2nd edn., Cambridge Studies in Advanced Mathematics, Cambridge University Press, Cambridge, 2001.

14. J. Milnor, On the Betti numbers of real varieties, Proc. Amer. Math. Soc., 15 (1964), 275-280.

15. O. A. Oleinik and I. B. Petrovskii, On the topology of real algebraic surfaces, Izv. Akad. Nauk SSSR, 13 (1949), 389-402.

16. P. Orlik and H. Terao, Arrangements of Hyperplanes, Springer-Verlag, New York, 1992. 
17. J. Rotman, An Introduction to Algebraic Topology, Springer-Verlag, New York, 1988.

18. M. Sharir, Arrangements of surfaces in higher dimensions, in Advances in Discrete and Computational Geometry, pp. 335-354, American Mathematical Society, Providence, RI, Contemporary Mathematics, Vol. 223, 1999.

19. R. Thom, Sur l'homologie des varietes algebriques reelles, Differential and Combinatorial Topology, pp. 255-265, S. S. Cairns (ed.), Princeton University Press, Princeton, NJ, 1965.

Received June 6, 2001, and in revised form June 19, 2001. Online publication May 7, 2003. 